Physical examination showed a man fairly well developed and very well nourished, distinctly apathetic. Speech was slow and uncertain, and mentality obviously dull. There were marked coarse, convulsive movements of extremities and tremor in hands. Right pupil was twice the size of left; both were regular, and responded to light and distance. Kneejerks were present, the right increased. Elbow-jerk was present on right side only. There was no clonus or Babinski, and no Romberg. Physical examination was otherwise negative. Wassermann of both blood and spinal fluid was positive.

Patient has had $4.5 \mathrm{gm}$. of neosalvarsan intravenously in five injections within three months and 60 c.c. of his own salvarsanized serum according to the technic previously outlined. Twice he has had mild reactions consisting of an exaggeration of the tremor and convulsive movements. There has been no other reaction. He has improved markedly so that he returned to work after the third injection and considers himself well. His wife notices, however, occasional evidences of mental impairment.

CASE 2 (No. 881). -Woman, aged 55, married, with nothing remarkable in her family or past history, for several years has suffered with varied "nervous manifestations"--diplopia intermittent in character, numbness and paresthesias of right side, transient right-sided hemiplegia, subsequent motor weakness, oecipital headaches, and finally two hard tumors of the skull. Wassermaun one year before had been negative, probably because of mercurial treatment.

Physical examination showed marked internal strabismus of right eye, motor and sensory impairment of right side and two firm tumors several centimeters in diameter on skull in frontal and temporal regions of right side. Wasscrmann on blood was repeated and found positive.

Patient received three intravenous injections of $0.6,0.9$ and $0.9 \mathrm{gm}$, respectively, of neosalvarsan, the second being followed by an intraspinal injection of 12 c.c. of her salvarsan. ized serum. Marked febrile reaction with chills followed the intravenous injections and severe backache, with transient increase of motor weakness, and headache followed the intraspinal injection.

Nodules on skull have disappeared, vision is greatly improved, and sensory and motor paresis are much less marked.

Case 3 (No. 939).-Man, aged 44, married, childless, with family and past histories not remarkable, denyiny venereal disease, for two and a half years has been troubled by twitching of cheeks and lips, "stoppage of talk," loss of memory, and impaired locomotion. Speech is incoherent and disconnected. Patient has grandiose manner and mild delusions of same type. Diagnosis of lues had been made and patient had received from twelve to fourteen injections of salvarsan and neosalvarsan intravenously with some improvement.

Physical examination revealed nothing remarkable beyond what has been noted in the history except increased kneejerks. Wassermann in the blood was negative and in the spinal fluid positive.

Patient has received $3.6 \mathrm{gm}$. of neosalvarsan intravenously in two months and 45 c.e. of his own salvarsanized serum intraspinally in four treatments without any reaction. His condition is greatly improved, the mental symptoms alone persisting though in lessened degree.

CASE 4 (No. 963).-Man, aged 39, married, childless, with family history and past history not remarkable, who had gonorrhea but denied luetic infection, for two years had been known to have tabes and gonorrheal prostatitis, but had been able to attend to his business. Three weeks before, he began to show signs of mental disturbance-was distinetly melancholy and apathetic. Two weeks before, after a hard day's work, he developed a mild mania with fixed delusions. There was incontinence of urine.

Physical examination revealed the usual signs of tabes. Wassermann was positive in both blood and spinal fluid.

Patient was given $2.7 \mathrm{gm}$. of neosalvarsan intravenously and 28 c.c. of his own salvarsanized serum intraspinally in three treatments within a period of seven weeks. Marked psychic reaction followed the first injection but no other reaction was observed.
Patient's condition las improved in every way. Recently he had the best days in two years, but is still irritational at times. Locomotion is very good.

CASE 5 (No. 949).-Man, aged 26, married, childless, with family history unimportant, admitting gonorrhea seven years ago and syphilis five years ago, suffered right hemiplegia six months ago. This improved for a time under treatment, but is now growing worse. Physical examination reveals only the hemiplegia. Wassermann of blood and spinal fluid is positive.

Patient received $2.4 \mathrm{gm}$. of neosalvarsan intravenously in three injections and 24 c.c. of his own salvarsanized serum in two intraspinal injections following the first two intravenous treatments. There was marked reaction causing increase of motor impairment of right side and some mental confusion after the intraspinal injections.

Patient has gained markedly in weight, speech has improved and locomotion has somewhat improved. He feels much better than at any time in last six months. He has acquired a fresh gonorrhea, or is suffering from a recrudescence of his former infection.

CAse 6 (No. 961).-Woman, aged 58, married, mother of eleven children, with family and past histories not remarkable, for last eight months has noticed "numbness" of hands and fect, followed by marked loss of strength. Vision and hearing are impaired, dizziness, headache and nausea are present at times. Coordination is poor. She cannot walk.

Physical examination shows normal pupils. moderate Romberg, paresthcsias but no anesthesias of arms and legs, kneejerks present and equal, and no clonus or Babinski. Wassermann positive in blool and negative in spinal fluid. (Only 0.1 c.e. was used.) Urinalysis reveals a chronic nephritis.

Patient received $0.8 \mathrm{gm}$. of neosalvarsan intravenously and 24 c.c. of serum intraspinally in two injertions. Mild reaction consisting of backache and nausea followed the first injection. Marked nausea of a week's duration but only mild backache followed the second.

P'atient's sensory impairment has but slightly improved. Locomotion is much less difficult.

Case 7 (No. 63).-Man, aged 38, unmarried, denying any knowledge of syphilitic infection, for two years has had minor epileptic seizures gradually increasing in number up to from eight to twelve daily.

Physical examination revealed nothing remarkable. Patient had improved on bromids, but only for comparatively short periods. Wassermann of blood proved positive, of spinal fluid (0.1 c.e.) negative.

Patient received $1.8 \mathrm{gm}$, of neosalvarsan intravenously anr 24 c.c. of salvarsanized serum intraspinally in two treatments. Temperature rose to 101,3 following first injection and patient complained of severe backache and occipital headache. Reaction following second injection was of same character bat much less intense.

Number of seizures has been diminished to one slight attark daily with several successive days of complete freedom at times. General condition has distinctly improved. Patient volunteers statement that he feels better than for years.

\section{PIRESENT-DAY DANGER OF ROENTGEN-RAY BURNS AND HOW TO PREVENT THEM}

\section{G. E. PFAHLER, M.D. PHILADELPHIA}

The serious Roentgen burns that were producel during the early years of roentgenology made both patients and physicians cautious, and during the last eight or ten years the work has been done almost exclusively by specialists who have devoted their entire time and attention to roentgenology, and who have learned how to avoid burns, and yet to produce good results.

With rare exceptions during the past ten years, we have heard of no burns of patients, and the only thing that has made us remember any danger of Roentgen 
rays is the announcement of the death, from time to time, of some roentgenologist who had damaged himself early in the work.

'I hie facts that these Roentgen ulcers in patients were produced during the early years of roentgenology, and that the damage done to roentgenologists occurred during these early years, have led the general profession to believe that danger in roentgenology has been eliminated, and that to-clay every one and any one can use the Roentgen rays with safety. This is true only so far as those using the Roentgen rays have acquainted themselves with the clangers and learned to avoid them. A razor is a dangerous instrument in the hands of a child, but perfectly safe in the hands of those skilled in its use.

The marvelous advances that have been made in roentgenology, and the important field that it occupies to-day in diagnosis and treatment, have led to the exploitation of apparatus among the general profession by manufacturers who for the most part have in mind only sales, and therefore leave the physician under the impression that all he needs is the apparatus to do good work, and do it safely.

It is to be feared that the combination of enthusiasm for the use of the Roentgen rays and the false sense of safety will lead to disastrous results in the hands of untrained and unguarded physicians. Therefore it seems timely that a note of warning be sounded, for already there lave come to my attention a number of serious Roentgen-ray burns produced recently by unskilled men, during single examinations. If this continues, much harm will be done to patients and physicians alike, and, secondarily, the specialty of roentgenology will be injured and its progress halted. It is a fact to-day that the Roentgen rays are absolutely harmless when properly used; that examinations can be made without the slightest injury to the patient, and that a roentgenologist probably can protect himself completely against its continuous and insidious evil influence. It is also a fact that the Roentgen rays as such are many times more dangerons to-day than they were ten years ago, because many times more powerful, and because the apparatus to-day is capable of a much larger output in a given time. 'T'en years ago, examinations were made with a current of from 1 to 5 milliamperes. To-day practically all examinations are made with from 10 to 100 milliamperes. Therefore it is easily seen that rays, as such, are ten times more dangerous to-day than they were ten years ago. They are made safe only because there is perhaps more than ten times as much knowledge concerning their use and dangers. This. knowledge is at hand, is the property of the profession and is open to those who are willing to take the trouble to enlighten themselves. Besides the actual statement of facts sucl as can be found in literature, a certain amount of skill is needed, which can only be obtained by actual training or experience.

\section{THE CALSE OF ROENTGEN-RAY BURNS}

The effect of the Roentgen rays on the tissues of the body depends on the quantity of rays absorbed, and the degree of sensibility of the tissues affected. The rays that pass through a tissue have practically no effect on it. Therefore it is the rays which are absorbed and do not pass through, which produce any beneficial or evil effect on the tissue. Therefore, the first essential is to be able to produce and judge the quality of the rays that will pass through a tissue. Most burns have been produced by the physician who lacks the ability to judge the penctration of the rays needed. As a result he is apt to use rass that are practically all absorbed in the superficial sofi tissue, producing varying degrees of burns, depending on the quantity of ravs. If he is using one of the modern powerful transformers, he can in a very short time produce enough rays to damage the soft tissucs. For instance, one of the worst burns I have ever seen was produced by a physician while using one of our modern equipments, in an attempt to make an cxamination for stone in the kidney. His exposure was only two and a half minutes, and in this time he obtained no shadows on his plate. When the patient was seen five months after this injury, there remained an ulcer about 5 inches in diameter, which was excruciatingly painful and was showing no tendency to heal. In the hands of a skilful operator, with the same apparatus, a good picture of the kidney would have been obtained in from three to five seconds, and with absolutely no harm.

'The damaging effects on patients and operators have been avoided in great part also during the past ten years, by the elimination of roentgenoscopic work.

The remarkable advances that have been made in the diagnosis of gastro-intestinal conditions by means of roentgenoscopy during the past five years have created a wave of enthisiasm for this class of work, and has led many into the use of the Roentgen rays for this purpose even though they do not take the trouble to make roentgenograms. This class of physicians is likewise apt not to take the trouble to learn the underlying principles governing the use of the Roentgen rays, and as a result they are apt to think that because they do not see a thing well, they are not using quite enough current. Current is, therefore, crowded into their tube, and while they do see a little better for the time being, they often produce a tremendous amount of soft rays, which are being absorbed by the soft tissues of the patients and themselves, which result in a dermatitis in both the patient and the operator. An illustration of this came to my attention recently. Two physicians were examining a forearm for a foreign body by moans of the roentgenoscope. The single examination was folloved by a well-marked dermatitis on the hands and forearms of the two physicians, and a Roentgen ulcer on the forearm of the patient that is not yet healed, several months after the injury. In the hands of a skilful operator a foreign body such as this can be localized and removed under the direction of roentgenoscopy, and do neither the patient nor the operator harm.

In roentgenoscopy it is not necessary to use a great amount of current. Most of my roentgenoseopy is done with 1 milliampere. In all roentgenoscopy both the time and the quantity of current should be kept at the minimum.

\section{HOW 'TO AYOID ROENTGEN BURNS DURING} EXAMINATIONS

In the aroidance of burns one must first keep in mind the principles governing the cause of the burns:

1. To use as small a quantity of rays as is consistent with the examination.

2 . To use a quality of rays that will penetrate the tissues, and not be entirely absorbed by the soft tissues. One can learn to judge the penetration by frequent use of the penetrometer and by the making of many roentgenograms. 
3. To make every examination as short as is possible, thereby lessening the total amount of rays to be absorbed.

4. To use intensifying screens when practicable.

5. To use filters for the elimination of the softer rays.

6. To confine the rays to the part actually under examination.

Burns of the operator may be avoided: (1) by keeping entirelv out of the field of rays, by working from an adjoining room, with lead-lined walls between, or by the use of lead-lined cabinets; (2) by confining the rays about the tube so that the only way of exit is through the aperture made for the examination of the patient; (3) by means of protecting shields, aprons, gloves, masks, etc. In fact, all these should be combined.

Burns during Roentgen therapy may be aroided, (1) by following the same general principles referred to in diagnosis; (2) by measuring each dose given and never exceeding the limit of skin toleration as indicated by the dose meter; (3) by allowing an interval of three weeks between the repetitions of the dose on any particular area of skin; (4) by the use of more filtration than would be used in diagnostic work; (5) by keeping in mind the fact that epithelium and glandular tissue are more sensitive than other tissue to the rays; $(6)$ bv avoiding any other form of irritation on the skin treated, such as counterirritation, high-frequency currents, liniments, stimulating ointments, antiseptics, etc.

The details necessary in carrying out the foregoing principles will vary with the individual operator and his circumstances, but they should receive the most careful attention. I believe that roentgenology is more distinctly a specialty than any other, because to master it one must be a good physician, must have a good general knowledge of pathology both in general medicine and the specialties, must have a large equipment, must give much time to the mastery of details, and must always be cautious.

1321 Spruce Street.

\section{TREATMENT OF TETANUS BY THE "RATIONAL" METHOD OF ASHHURS'T AND JOHN}

THE DFVELOPMENT OF SUPPURATIVE SERUM (ASEPTIC) MENINGITIS FOLLOWING THE INTRASPINAL INJECTION OF TETANUS ANTITOXIN; WITH REPORT OF A CASE *

\section{WALTER V. BREM, M.D. LOS ANGELES}

In a recent article on the treatment of tetanus, Ashhurst and John ${ }^{1}$ conclude that the rational use of tetanus antitoxin consists in (1) the intraneural injection of antitoxin; (2) the intraspinal injection; (3) the intravenous injection, and (4) the infiltration of the tissues about the site of the injury.

The quantity used.should be very much greater than the quantity that has usually been given heretofore by the subcutaneous route. In one of their cases in which the subcutaneous route was used, 224,000 units were

* Read at the menting of the Los Angeles County Medical Association, Oct. 16, 1913 .

1. Ashhurst, A. P. C., and John, R. L. : The Rational Treatment if. administered in three days. But such an enormous quantity is not demanded when the intraneural and intraspinal routes are chosen. These authors feel that the outlook for patients with tetanus is much brighter now since the development of this method of administering antitoxin, and that the old view, that antitoxin is of no avail after the symptoms of tetanus have developed, must be abandoned.

In 1910, at Colon Hospital, Canal Zone, I treated four cases of tetanus by intraneural, intravenous and subcutaneous injections of antitoxin, and used chlorbutanol (chloretone) and morphin as sedatives. These cases are reported in detail by Hill. ${ }^{2}$

One of these patients, whose case was a severe one with a ten-day incubation period, recovered. From this patient the infecting tetanus bacillus was passed through a series of guinea-pigs. 'The last two of these pigs, after local tetanus was well developed, were treated by postorbital injection of 1 c.c. of tetanus antitoxin. One pig recovered, and the other seemed to be recovering when it was accidentally killed by a subcutaneous injection of chlorbutanol in alcohol. Smears made from the wounds showed Gram-positive bacilli that appeared to be in all stages of degeneration. This was shown by great swelling and granulation of the bacilli, atvoical shapes, and the absence of spore formation. Furthermore, the splinter which had been removed from the patient's wound and had been used in successive inoculations, failed to infect another pig when removed from the last-treated animal. The dosage used in treating these animals postorbitally was equivalent to about 30,000 units in an adult man.

I. have not had an opportunity since then to examine the results that I obtained in the treatment of these guinea-pigs, but I felt the results suggestive enough to warrant the use of intraspinal injections in future cases of tetanus. I was ready, therefore, to accept the views of Ashhurst and John when their article appeared, and shortly afterward I had an opportunity to apply, successfully, their methods to the treatment of a patient with cephalic tetanus, which developed after a six-day incubation period.

\section{REPORT OF CASE}

Injury by baseball on upper lip; six-day incubation period; gradual onset; intraneural, intraspinal and intravenous injection of 98,000 units of tetanus antitoxin and infiltration of lip about the injury; suppurative serum (aseptic) meningitis follouing intraspinal injection of antitoxin; rapid rocovery.

The case occurred in the private practice of Dr. P. Newmark of Los Angeles, who called me in consultation and requested me to treat the patient. I wish to thank Dr. Newmark here for permitting me to publish the case-report, and for his courtesy and encouragement during the trying days of the treatment.

History.-The patient was a robust man aged 2l. He had been struck on the left side of the upper lip by a baseball on Saturday, July 12, 1913. The blow caused an abrasion of the skin, and the mucous surface of the lip was cut by the impact against a tooth. The skin abrasion was treated with hydrogen peroxid by the patient's mother several hours after the injury occurred. Healing of the wounds, both inside and outside, was rapid. On Friday evening, July 18, the patient's teeth clamped down tightly on a cigar that he had begun to smoke, and he noticed that his jaws were stiff. His jaws were stiffer on Saturday, the 19th, and he began to feel pain in his back. He called on Dr. Newmark on this date, but not finding him he did not see him until Sunday afternoon, July 20 , when the back was more painful and was stiff and the jaws were about as stiff as on the day before. The patient

2. Hill, E. W. : Tetanus, Arch. Int. Med., Dec., 1911, p. 747. 